\title{
Pregnancy outcome of women presenting with decreased fetal movements
}

\begin{abstract}
Objective: To evaluate and analyze pregnancy and neonatal outcome in a group of pregnant women who were hospitalized due to decreased fetal movements (DFM).

Methods: This was a retrospective case control study of 70 pregnant women with the "Decreased fetal movements ante partum" diagnosis who were hospitalized, induced and delivered following the same event. This group was compared to 70 other pregnant women who were hospitalized due to the same diagnosis, evaluated, discharged home and delivered at our center later on. These 2 DFM groups were compared to a control group.
\end{abstract}

Results: During the study period, 17,465 women delivered at our medical center, 2,366 women hospitalized in our high-risk unit, including 171 having DFM as their primary diagnosis. Compared to the DFM group who was discharged home and the control group, the induced DFM group was at higher gestational age at hospital admission, had higher "high risk pregnancy" and "poor pregnancy outcome" rates, as well as higher cesarean section (CS), neonatal intensive care unit NICU admission and hospitalization period rates.

Conclusions: Pregnant women who complain of DFM at term are more prevalent as higher risk pregnancy, Poor pregnancy outcome; more often labor induced have and higher CS rates.
Volume 9 Issue 4 - 2018

\author{
Yuri Perlitz,' Izhar Ben-Shlomo,' Johnny \\ Younis,' Moshe Ben-Ami,' Reut Badihi \\ 'Department of Obstetrics \& Gynecology, Baruch Padeh \\ Medical Center, Israel \\ ${ }^{2}$ Medical student, Azrieli Faculty of Medicine, Bar-llan University, \\ Israel
}

Correspondence: Yuri Perlitz, Department of Obstetrics \& Gynecology, Baruch Padeh Medical Center, Poriya, Azrieli Faculty of Medicine, Bar-llan University, Safed, MPO Lower Galilee, 15208, Israel, Tel +972-4-6652309, Fax +972-4-6652487, Email yperlitz@poria.health.gov.il

Received: June 02, 2018 | Published: July 13, 2018

\section{Introduction}

Routine fetal movements (FM) counting is a common practice in pregnancy, used to verify fetal well-being. ${ }^{1}$ FM counting is practiced beginning at 24-26 weeks' gestation until delivery. ${ }^{2}$ There are no agreed guidelines concerning the number and frequency of perceived movements needed for neither fetal health reassurance, nor management options when a pregnant woman complains of decreased fetal movements (DFM). ${ }^{3}$ However, most pregnant women are encouraged by their caregivers to face medical assistance when they have a DFM episode. ${ }^{4}$ The next steps for evaluation of fetal wellbeing in these cases include non-stress test (NST) and biophysical profile (BPP). Estimated fetal weight (EFW) and Doppler assessment of the umbilical and middle cerebral arteries are assessed when restriction of growth is suspected. ${ }^{4,5}$ Even when we detect no fetal/ maternal compromise or pathology (i.e. placental insufficiency, hypertension, diabetes, intra-uterine growth restriction) and the gravida is at term, we frequently offer her induction of labor, based on the assumption that we might have missed a pathology causing DFM. By performing the induction process, we do not jeopardize the patient or her fetus. ${ }^{6}$ However, this attitude may lead to longer than expected hospitalization, a need for cervical ripening or a need for induction of labor, all potentially leading to anxiety of the patient and her family. Is this approach justified? What may be its' consequences?

In this study, we aimed to evaluate and analyze pregnancy outcome in a group of pregnant women who were hospitalized due to DFM. Further analysis was performed comparing specifically the group of women who remained hospitalized and were induced, to those who were discharged home and delivered at our medical center later on.

\section{Materials and methods}

This was a retrospective case control study. We searched our highrisk unit admissions report for women who were hospitalized due to
DFM from January $1^{\text {st }} 2012$ to December $31^{\text {th }} 2016$. We identified these women by sampling the diagnosis: "Decreased fetal movements ante partum" (ICD9 code 655.73) as their primary diagnosis. The prevalence of women who suffers from DFM is approximately 5-15\%. Most of them are being examined by their physician and continue the pregnancy unharmed. ${ }^{7,8}$ The percentage of pregnancies induced due to DFM is unknown. So, we chose a convenient number of participants for this study. The first 70 pregnant women with the DFM diagnosis who were hospitalized and delivered following the same event (group A) and 70 other pregnant women who were hospitalized due to the same diagnosis, evaluated, discharged home and delivered at our medical center later on (group B). These 2 DFM groups were compared to a control group (group C). This group included the first 3 pregnant women who were hospitalized at our medical center each month during the study period who did not have the diagnosis "Decreased fetal movements ante partum" as part of their admission report (Figure1). In addition, this control group included only women who had no maternal, placental or fetal complications, and were admitted only for labor. The study was approved by the local Institutional Review Board number 0037-16-POR dated 11/08/2016. Our medical center is an academic, university-affiliated center. Our departmental protocol declares that when a pregnant woman addresses the obstetrics emergency room complaining of DFM, we assess the fetal well-being by performing NST and BPP. When we suspect intra uterine growth restriction (IUGR) we perform EFW and Doppler studies of the umbilical artery, middle cerebral artery (MCA) and calculate the Cerebro-Placental-Ratio (CPR). If all these tests are reassuring, together with no other maternal, obstetrical or fetal pathological factors, and the patient continues to complain of DFM, we postpone her discharge home and perform another NST 2-3 hours later. If the DFM complaints continue after that, we hospitalize these women overnight for further fetal surveillance. When the DFM complaints continue the next day, even with no maternal, obstetrical or fetal complications, and the pregnancy is at term, we offer these 
women induction of labor (IOL). We discharge home pregnant women if their DFM episode is resolved. We equip them with a "Fetal Movements Chart" containing instructions for time intervals, frequency of expected fetal movements and a recommendation to return for obstetrical examination if an episode of DFM recurs.

Statistical analysis tests used to analyze data in this study were ANOVA, T test and Chi square test, as applicable. For regression analysis, "pregnancy outcome" has been the dependent parameter, and the following were the independent parameters: maternal age, body mass index (BMI), gestational age at hospitalization, smoking, length of DFM episode, and the study groups. We used the term "poor outcome" in cases of one or more of the following events occurred (delivery by CS, APGAR score $<7$ at 5 minutes, cord $\mathrm{pH}<7.2$ or neonatal intensive care unit (NICU) admission). If none of these complications occurred, we classified these cases as "favorable outcome". The term "high risk pregnancy" (HRP) was used for any maternal/fetal complication, occurring before the DFM hospitalization, which necessitated special surveillance at the highrisk pregnancy Clinique of our medical center. $\mathrm{P}<0.05$ was considered significant for our data.

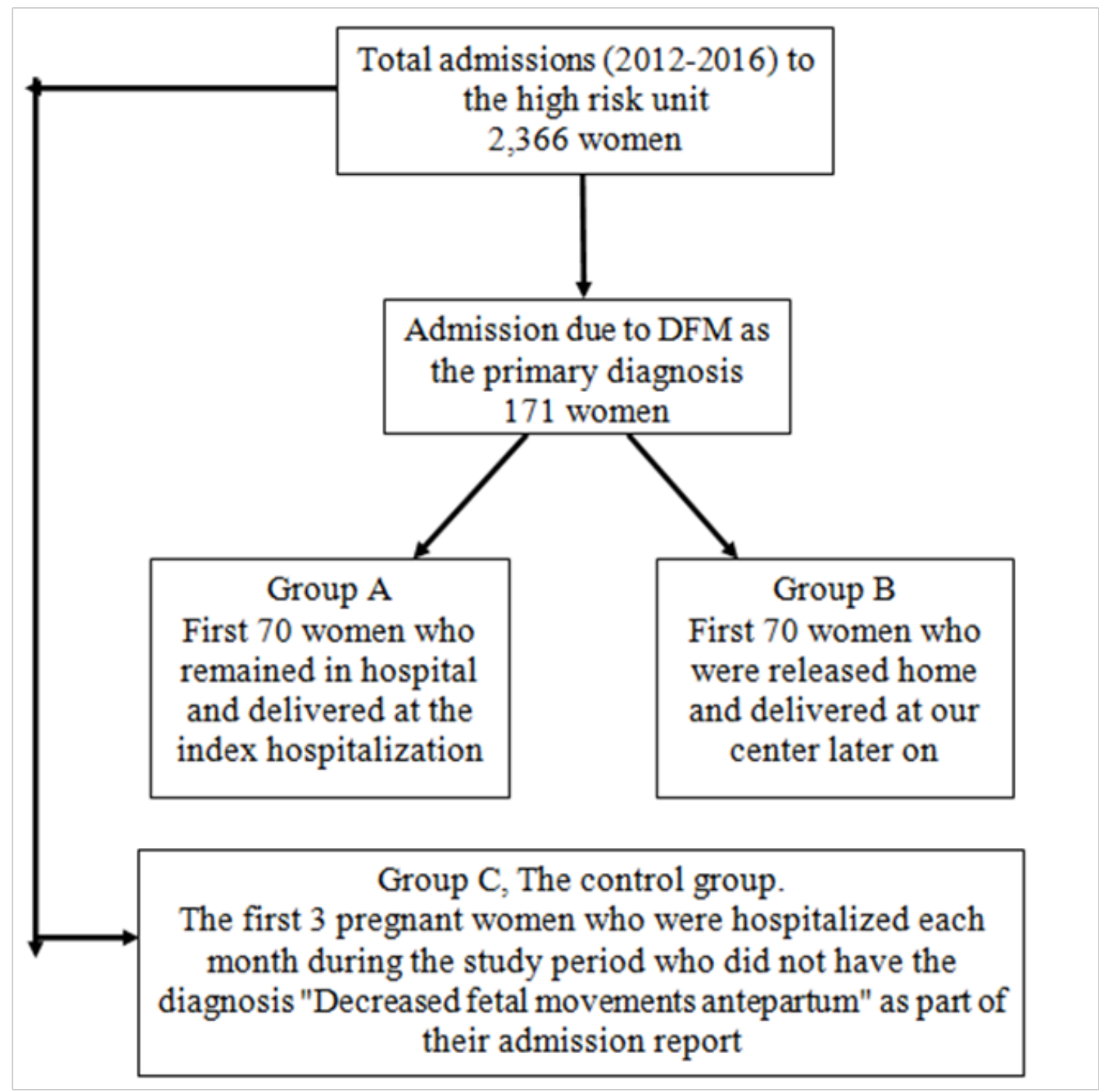

Figure I Flow chart of study and control groups.

\section{Results}

During the specified five years, 17,465 women delivered at our medical center, a mean of 3,493 deliveries per annum. During this period, 2,366 patients were hospitalized in our high-risk unit, including 171 having DFM as their primary diagnosis. Maternal and obstetrical results of DFM \& IOL (group A), DFM \& discharge (group B) and controls (group C) are presented in table 1. Delivery and neonatal results of the 3 study groups are presented in table 2 . We detected a significant difference in the gestational age at hospitalization between groups A (38.7 weeks) and B (35.4 weeks). Women who had DFM at earlier gestational age were released home more often than those at term. There was a significant difference between the groups in the rate of high-risk pregnancies. Sixty percent of women in group A were classified as "high risk pregnancy" in comparison to $22 \%$ in group B and $18 \%$ in group $\mathrm{C}\left(\chi^{2}=15.99, \mathrm{p}<0.05\right)$ (Table 1$)$. Mode of delivery was very different between groups $\mathrm{A}$ and $\mathrm{C}\left(\chi^{2}=58.27, \mathrm{p}<0.05\right.$ (. Fifty seven percent of women at group A delivered by CS (Table 2). NICU 
admissions were more prevalent in-group A than B and C (56\% vs. $33 \%$ vs. $11 \%$ ). Among women who suffered "poor outcome ", $80 \%$ belonged to group A $12 \%$ to group B and $8 \%$ to group C $\left(\chi^{2}=70.136\right.$, $\mathrm{p}<0.05$ ) (Table 2).

The following parameters were detected as significantly related to "poor outcome": earlier gestational age at admission to hospital and prolonged hospitalization of women in-group A. No fetal or neonatal mortality occurred in our study. Assessment of the data according to IOL vs. spontaneous start of labor (Table 3) revealed the following: The mean gestational age at admission was higher in the IOL group $(\mathrm{F}=2.5, \mathrm{p}<0.05)$.Duration of hospitalization was significantly higher in the IOL group $(\mathrm{F}=2.0, \mathrm{p}<0.05)$.

Table I Maternal and obstetrical results of DFM\&IOL (group A), DFM \& discharge (group B) and controls (group C)

\begin{tabular}{|c|c|c|c|c|c|c|}
\hline Variable & Category & $\begin{array}{l}\text { DFM \& IOL } \\
\text { (Group A) }\end{array}$ & $\begin{array}{l}\text { DFM \& } \\
\text { discharge } \\
\text { (Group B) }\end{array}$ & $\begin{array}{l}\text { Controls } \\
\text { (Group C) }\end{array}$ & $\mathbf{p}$ & Test \\
\hline \multirow[b]{2}{*}{ Age } & \multirow[b]{2}{*}{ Participants } & 70 & 70 & 70 & \multirow[b]{2}{*}{0.371} & Anova \\
\hline & & $29.1 \pm 5.5$ & $28.1 \pm 5.1$ & $29.2 \pm 5.2$ & & Mean \pm SD \\
\hline \multirow[t]{2}{*}{ Gestational age at admission } & & $38.7 \pm 1.4$ & $35.5 \pm 4.0$ & $39.6 \pm 1.1$ & \multirow[t]{2}{*}{0.00} & \\
\hline & & & & & & Mean \pm SD \\
\hline \multirow{2}{*}{ Smoking } & Yes (\%) & $5(46)$ & $4(36)$ & $2(18)$ & \multirow{2}{*}{0.38} & \multirow{2}{*}{ Chi square } \\
\hline & Unknown (\%) & $20(42)$ & $12(25)$ & $16(33)$ & & \\
\hline Nulliparity (\%) & & $28(33)$ & $39(46)$ & $17(20)$ & 0.00 & Chi square \\
\hline High Risk Pregnancy (\%) & & $42(60)$ & $15(22)$ & $13(18)$ & 0.00 & Chi square \\
\hline \multirow{2}{*}{ Maternal BMI } & \multirow{2}{*}{ Participants } & 65 & 49 & 66 & \multirow{2}{*}{0.06} & Anova \\
\hline & & $29.6 \pm 4.2$ & $29.8 \pm 5.6$ & $29.1 \pm 4.6$ & & Mean \pm SD \\
\hline
\end{tabular}

$\mathrm{IOL}$, induction of labor; BMI, Body mass index; SD, standard deviation

Table 2 Mode of delivery and pregnancy outcome of DFM and control groups

\begin{tabular}{llllll}
\hline & DFM Delivered & DFM Released & Controls & P & Test \\
\hline Number & $70(\%)$ & $70(\%)$ & $70(\%)$ & & \\
Mode of delivery & & & & \\
Spon.Vag: & $24(35)$ & $62(89)$ & $67(96)$ & & \\
Operative: & $6(8)$ & $3(4)$ & $1(1)$ & 0.00 & Chi square \\
C/S: & $40(57)$ & $5(7)$ & $2(3)$ & & \\
Birth weight (g) & & & & \\
Mean \pm SD & $3123 \pm 477$ & $3220 \pm 507$ & $3369 \pm 394$ & 0.00 & ANOVA \\
5 min APGAR score & & & & & \\
Mean \pm SD & $8.7 \pm 0.99$ & $8.9 \pm 0.91$ & $9.35 \pm 0.59$ & 0.00 & ANOVA \\
Cord pH & & & & & \\
$\begin{array}{l}\text { Mean } \pm \text { SD } \\
\text { Duration of hospitalization }\end{array}$ & $7.26 \pm 0.1$ & $7.29 \pm 0.1$ & $7.29 \pm 0.03$ & 0.38 & ANOVA \\
Mean \pm SD & $4.89 \pm 1.7$ & $3.2 \pm 1.3$ & $3.1 \pm 2.3$ & 0.00 & T test \\
NICU hospitalization & $10(56)$ & $6(33)$ & $2(11)$ & 0.05 & Chi square \\
Poor pregnancy outcome & $42(80)$ & $6(12)$ & $4(8)$ & 0.00 & Chi square \\
\hline
\end{tabular}

DFM, decreased fetal movements; NICU, neonatal intensive care unit; C/S, Cesarean section; Spon.Vag, Spontaneous vaginal 
Table 3 Outcomes of the DFM groups $(A+B)$ according to induction of labor/ spontaneous labor start

\begin{tabular}{|c|c|c|c|c|c|}
\hline Number & IOL 62 & Spont. 78 & $\mathbf{F}$ & $\mathbf{p}$ & Test \\
\hline \multicolumn{6}{|c|}{ Maternal age (Years) } \\
\hline Mean \pm SD & $28.8 \pm 5.3$ & $29.3 \pm 5.3$ & -0.5 & 0.6 & $T$ test \\
\hline \multicolumn{6}{|c|}{ Gestational age at admission } \\
\hline Mean \pm SD & $39.5 \pm 1.3$ & $38.9 \pm 1.5$ & 2.5 & 0.01 & ANOVA \\
\hline \multicolumn{6}{|c|}{ Maternal BMI } \\
\hline Mean \pm SD & $29.6 \pm 4.8$ & $28.1 \pm 4.1$ & 1.9 & 0.06 & ANOVA \\
\hline \multicolumn{6}{|c|}{ Birth weight (g) } \\
\hline Mean \pm SD & $\begin{array}{l}3306 \pm \\
410\end{array}$ & $\begin{array}{l}3197 \pm \\
482\end{array}$ & 1.4 & 0.23 & $T$ test \\
\hline \multicolumn{6}{|c|}{5 min APGAR score } \\
\hline Mean \pm SD & $8.9 \pm 0.9$ & $9.1 \pm 0.8$ & -0.8 & 0.4 & ANOVA \\
\hline \multicolumn{6}{|c|}{ Duration of hospitalization (days) } \\
\hline Mean \pm SD & $4.4 \pm 2.6$ & $3.6 \pm 1.8$ & 2 & 0.04 & $T$ test \\
\hline
\end{tabular}

BMI, body mass index $\left(\mathrm{kg} / \mathrm{m}^{2}\right)$; IOL, induction of labor

\section{Discussion}

In this study, we evaluated and analyzed pregnancy outcome of women who were hospitalized due to DFM. We further divided this group to a group were labor was induced, or after having reassurance of the baby, women were sent home and delivered at our center later on. The DFM group who was induced was statistically at higher gestational age and "high risk pregnancy" (HRP) compared to the women in the group who were discharged home. Our results are in accordance with the recommendations of Unterscheider et al. ${ }^{9}$ who suggested a similar management protocol for patients who presented with DFM, including IOL if DFM persists and the pregnancy is at term. Furthermore, the 2 parameters (term gestational age and HRP in addition to DFM) are only logical and self-explanatory for the higher induction rate of this group. Number of hospitalization days was higher at the DFM \& IOL group than the control group. It is the result of the ripening and induction processes of the DFM \& IOL group, which takes more time than spontaneous non-induced labor as most of the controls. High percentage of DFM \& IOL group women delivered by CS. This finding is in line with the fact that this group had more cases of poor outcome (Group A - 80\%, group B $-12 \%$, group C 8\%). Our results are in accordance with Nor Azlin et al. ${ }^{10}$ study, who analyzed 230 cases of women who presented primarily with reduced fetal movements. Less than half (49\%) of the women had spontaneous labor, $48 \%$ had induction and $6 \%$ had elective caesarean section. Aviram et al. ${ }^{11}$ analyzed pregnancy outcome in women with decreased fetal movements according to parity. The authors of this study concluded that DFM is associated with increased risk of IOL $(20 \%)$ Vs. $8 \%$ in a control group. Furthermore, cesarean delivery and immediate increased risk for adverse perinatal outcome were detected in the DFM group. In the $2^{\text {nd }}$ step of our study, we analyzed the DFM 2 groups for maternal, obstetrical, delivery and neonatal outcome according to IOL or spontaneous start of labor. We noticed 2 significant differences: 1) the gestational age at admission to hospital was higher in the group who started the delivery spontaneously. It matches very well our findings of the DFM \& IOL group being more "high risk" and having more cases of "poor outcome", necessitating IOL earlier than the DFM \& discharge group. 2) Duration of hospitalization was higher in the IOL group. Obviously, it happened because it took more time to ripen the cervix and induce labor than a spontaneous delivery. For decades, IOL was believed to increase CS rate and other maternal complications. During recent years, well-performed studies contradicted this belief. The "35/39 study" concluded that among women of advanced maternal age, IOL at 39 weeks gestation, as compared to expectant management, had no significant effect on the rate of CS and no adverse short-term effects on maternal or neonatal outcomes. ${ }^{12}$ Miller et al. ${ }^{13}$ compared IOL of a group of nulliparous women with an unfavorable cervix with an expectant management group. The elective IOL group had cesarean delivery rate not doubled. Darney and colleges compared elective induction at each term gestational age (37-40 weeks) with expectant management in vertex, nonanomalous, singleton deliveries. They revealed the odds of cesarean delivery were lower among women with elective induction compared with expectant management across all gestational ages and parity. ${ }^{14}$ In a recent Cochrane review women with term premature rupture of labor were induced and compared to a similar group who was managed by expectant management. Women who performed IOL had less maternal/neonatal infection rate. No clear differences between the IOL and expectant management groups were seen for the risk of CS (average RR 0.84 ; $95 \%$ CI 0.69 to 1.04). ${ }^{15}$ Boulvain et al. ${ }^{16}$ compared induction of labor with expectant management for large-for-date fetuses for neonatal and maternal morbidity associated with macrosomia. They concluded that Induction of labor for suspected large-for-date fetuses is associated with a reduced risk of shoulder dystocia and associated morbidity compared with expectant management. Induction of labor did not increase the risk of caesarean delivery and improved the likelihood of spontaneous vaginal delivery. Our study's limitations are mainly its' retrospective design and small number of participants, making it impossible to evaluate rare fetal/neonatal complications, as well as maternal morbidities. Because of the retrospective nature of our study and the study design, we could not answer the question whether IOL is safer for the baby and the mother.

\section{Summary}

Our study revealed that a DFM complaint during pregnancy is more prevalent in "high-risk pregnancies" and is a risk factor for "poor pregnancy outcome" both for the mother and the neonate. Furthermore, high percentage of the group who were induced delivered by CS. Additional large, prospective, randomized trials are necessary for establishing the fetal/neonatal/maternal outcome associated with DFM, and to investigate the best management approach of this issue.

\section{Acknowledgments}

None.

\section{Conflict of interest}

The authors declare that they have no conflict of interest.

\section{References}

1. Sadovsky E, Yaffe H. Daily fetal movements recording and fetal prognosis. J Obstet Gynecol. 1973;41(6):845-850.

2. Delaram M, Jafarzadeh L. The effects of fetal movement counting on pregnancy outcomes. J Clin Diagn Res. 2016;10(2):SC22-SC24.

3. O’Sullivan O, Stephen G, Martindale E, et al. Predicting poor perinatal outcome in women who present with decreased fetal movements. J Obstet Gynaecol. 2009;29(8):705-710.

4. Practice Bulletin No. 145: Antepartum Fetal Surveillance. American College of Obstetrics and Gynecologists. 2014;124(1):182-192. 
5. Pearson JF, Weaver JB. Fetal activity and fetal well-being: an evaluation Br Med J. 1976;1(6021):1305-1307.

6. Little SE, Caughey AB. Induction of Labor and Cesarean: What is the true Relationship? Clin Obstet Gynecol. 2015;58(2):269-281.

7. Sergent F, Lefevre A, Verspyck E, et al. Decreased fetal movements in the third trimester: what to do? Gynecol Obstet Fertil. 2005;33(11):861-869.

8. Heazell AE, Green M, Wright C, et al. Midwives and obstetricians knowledge and management of women presenting with decreased fetal movements. Acta Obstet Gynecol Scand. 2008;87(3):331-339.

9. Unterscheider J, Horgan R, O’Donoghue K, et al. Reduced fetal movements. Obstetrician \& Gynecologist. 2009;11:245-251.

10. Nor Azlin MI, Maisarah AS, Rahana AR, et al. Pregnancy outcomes with a primary complaint of perception of reduced fetal movements. J Obste Gynaecol. 2015;35(1):13-15.

11. Aviram A, Shmueli A, Hiersch L, et al. Pregnancy Outcome in Women with Decreased Sensation of Fetal Movements at Term According to Parity. Birth. 2016;43(1):42-48.
12. Walker KF, Bugg GJ, Macpherson M, et al. Randomized trial of labor induction in women 35 years of age or older. $N$ Engl $\mathrm{J} \mathrm{Med}$. 2016;374(9):813-822.

13. Miller NR, Foglia LM, Pates JA, et al. Elective Induction of Labor Compared With Expectant Management of Nulliparous Women at 39 Weeks of Gestation: A Randomized Controlled Trial. Obstet Gynecol. 2015;126(6):1258-1264.

14. Darney BG, Snowden JM, Cheng YW, et al. Elective induction of labor at term compared with expectant management: maternal and neonatal outcomes. Obstet Gynecol. 2013;122(4):761-7699.

15. Middleton P, Shepherd E, Flenady V, et al. Is it better for a baby to be born immediately or to wait for labour to start spontaneously when waters break at or after 37 weeks? Cochrane Database of Systemic Reviews. 2017.

16. Boulvain M, Senat MV, Perrotin F, et al. Induction of labour versus expectant management for large-for-date fetuses: a randomized controlled trial. Lancet. 2015;385(9987):2600-2605. 